\title{
Ladder approximation in coupled quantum-well systems
}

\author{
A. Yurtsever and B. Tanatar \\ Department of Physics, Bilkent University, Bilkent, 06533 Ankara, Turkey
}

(Received 18 October 2000; published 12 March 2001)

\begin{abstract}
We study the contact values of the interlayer pair-correlation function in electron-electron and electron-hole double-layer systems. For this purpose the ladder approximation as generalized to multicomponent systems is used. The ladder approximation yields positive values for the interlayer $g_{\text {ee }}(0)$ and $g_{\text {eh }}(0)$ for all values of the density parameter $r_{s}$ and layer spacing $d$. This allows us to infer possible instabilities in the system more reliably compared to other approaches. We also investigate the effects of quantum-well width and screening on the interlayer pair-correlation functions.
\end{abstract}

DOI: 10.1103/PhysRevB.63.125320

PACS number(s): 73.63.Hs, 05.30.Fk, 71.45.Gm

\section{INTRODUCTION}

Double-layer electron-electron and electron-hole systems are under extensive theoretical and experimental study in recent years. ${ }^{1}$ Advances in growth techniques in semiconductors have led to the detailed investigations of multilayer structures. It is generally believed that in these systems the effects of interparticle interactions are enhanced because of reduced dimensionality and an extra degree of freedom provided by the layer index. Study of manybody effects ${ }^{2}$ in lowdimensional electronic systems, thus, became amenable to experimental observations.

In this work we apply the ladder theory to double-layer electron-electron and electron-hole systems. Our broad aim is to see how the effective interactions treated within the ladder approximation compare with other theoretical approaches, such as the self-consistent field approach. In particular, we are motivated by the recent work of Liu, Świerkowski, and Neilson ${ }^{3}$ who studied the exciton formation in spatially separated electron-hole liquids based on the Singwi, Tosi, Land, and Sjölander (STLS) formalism. ${ }^{4}$ It has been known that the self-consistent field method of STLS applied to multicomponent systems yields pair-correlation functions that are at quantitative and qualitative variance with other theoretical approaches. 5,6 Therefore, our aim is to study the interlayer pair-correlation functions at contact $g_{\text {ee }}(0)$ and $g_{\text {eh }}(0)$ for electron-electron and electron-hole double-layer systems.

The ladder approximation was introduced for the purpose of studying the pair-correlation function in electron gas systems. ${ }^{7}$ There has been many detailed studies in a variety of contexts and dimensionalities making use of the particleparticle ladder vertex function in the past. ${ }^{8-13}$ Freeman $^{14}$ studied the correlation energy and antiparallel spin paircorrelation function of the two-dimensional electron gas within the particle-particle approximation of the coupledcluster equations making contact to the ladder approximation. Recently, an extension of the ladder theory to multicomponent systems were given in a series of papers by Vericat and Melgarejo, ${ }^{15}$ Pugnaloni, Melgarejo, and Vericat $^{16}$ and Melgarejo et al. ${ }^{17}$ in connection with photoexcited electron-hole systems in quantum wells and quantum wires. We employ the multicomponent generalization of the ladder theory in application to double-layer electron-electron and electron-hole systems and particularly calculate the contact values of the pair-correlation function for these systems. The divergence in the interlayer $g_{\text {eh }}(0)$ may be used to understand the excitonic instability building in a spatially separated electron-hole system.

The rest of this paper is organized as follows. In Sec. II we introduce the formalism of ladder approximation for double-layer systems. In Sec. III we present our results for electron-electron and electron-hole double-layers considering also the effects of screening. Our results are discussed in relation to other theoretical approaches in the same section. Finally, we conclude with a brief summary in Sec. IV.

\section{MODEL AND THEORY}

We choose a simple model of double-layer system in the form of a pair of quantum-wells separated by an infinite barrier. The intra- and inter-layer Coulomb interactions are given by $V_{\alpha \beta}(q)=2 \pi e^{2} e^{-q d\left(1-\delta_{\alpha \beta}\right)} /\left(\epsilon_{0} q\right)$, in which $\epsilon_{0}$ is the background dielectric constant, $\alpha, \beta=1,2$ are the layer indices, and $d$ is the distance between the quantum wells. The effective interaction between two charge carriers within the ladder approximation is given by

$$
\begin{aligned}
I_{\alpha \beta}\left(k_{1}, k_{2} ; q\right)= & V_{\alpha \beta}(q)+\sum_{k} V_{\alpha \beta}(q-k) \\
& \times \frac{\left[1-f_{\alpha}\left(k_{1}+k\right)\right]\left[1-f_{\beta}\left(k_{2}-k\right)\right]}{\epsilon_{k_{1}, \alpha}-\epsilon_{k_{1}+k, \alpha}+\epsilon_{k_{2}, \beta}-\epsilon_{k_{2}-k, \beta}} \\
& \times I_{\alpha \beta}\left(k_{1}, k_{2} ; k\right),
\end{aligned}
$$

where $f_{\alpha}(k)$ is the zero-temperature Fermi distribution function and $\epsilon_{k, \alpha}=k^{2} / 2 m_{\alpha}$ is the single-particle energy for species $\alpha$. When the short-range correlations are assumed to be most important, we can neglect the dependence of $I_{\alpha \beta}\left(k_{1}, k_{2} ; q\right)$ on $k_{1}$ and $k_{2}$, and consider $I_{\alpha \beta}(q)$ $=I_{\alpha \beta}(0,0 ; q)$, which is a large momentum transfer $q$ approximation. Such an approximation was shown to be reasonable by full numerical solutions ${ }^{10,18}$ of the integral equation given in Eq. (1). Within this short-range approximation and for a double-layer system, the above integral equation simplifies to 


$$
\begin{aligned}
I_{\alpha \beta}(q) / V_{11}\left(k_{F}\right)= & \frac{e^{-\sqrt{2} q d\left(1-\delta_{\alpha \beta}\right) / a_{B}^{*} r_{s}}}{q}-\sqrt{\frac{2 r_{s}}{2 \pi}} \\
& \times \int_{1}^{\infty} d k \frac{F_{\alpha \beta}(q, k)}{k(q+k)} I_{\alpha \beta}(k) / V_{11}\left(k_{F}\right),
\end{aligned}
$$

where

$$
F_{\alpha \beta}(q, k)=\int_{0}^{\pi / 2} d \theta \frac{e^{-\sqrt{2} d\left[1-4 k q \sin ^{2} \theta /(q+k)^{2}\right]^{1 / 2}\left(1-\delta_{\alpha \beta}\right) / a_{B}^{*} r_{s}}}{\left[1-4 k q \sin ^{2} \theta /(q+k)^{2}\right]^{1 / 2}} .
$$

In the case of intralayer correlations $(\alpha=\beta), F_{\alpha \alpha}(q, k)$ reduces to $K(2 \sqrt{q k} /(q+k))$, where $K(x)$ is the complete elliptic integral of the first kind. In the above expression we have scaled wave vectors with the Fermi wave vector $k_{F}$ $=\sqrt{2 \pi^{2} n}$, where $n$ is the layer density. A convenient way of expressing the density is through the dimensionless parameter $r_{s}=1 / \sqrt{\pi n a_{B}^{* 2}}$, where the effective Bohr radius is defined as $a_{B}^{*}=\hbar^{2} / \mu_{\alpha \beta} e^{2}$. In the definition of $a_{B}^{*}$ we have used the reduced mass $1 / \mu_{\alpha \beta}=1 / m_{\alpha}+1 / m_{\beta}$.

The quantity of interest in detecting exciton formation in electron-hole systems is the interlayer contact paircorrelation function $g_{\text {eh }}(0)$. In a paramagnetic system $g_{\text {eh }}(r)$ is an arithmetic average of the spin-parallel $g_{\mathrm{eh}}^{\uparrow \uparrow}(r)$ and spinantiparallel $g_{\text {eh }}^{\uparrow \downarrow}(r)$ pair-distribution functions. For the interlayer pair-correlation function at contact we have $g_{\text {eh }}(0)$ $=g_{\text {eh }}^{\uparrow \downarrow}(0)$. The expression for $g_{\text {eh }}^{\uparrow \downarrow}(0)$ is obtained from the Goldstone formula for the energy shift, and within our approximation scheme it is given by

$$
\begin{aligned}
g_{\alpha \beta}^{\uparrow \downarrow}(r=0)= & {\left[1-\frac{\mu_{\alpha \beta}}{\pi} \int d^{2} q \frac{I_{\alpha \beta}(q)}{q^{2}}\right.} \\
& \left.\times \theta\left(q-k_{F \alpha}\right) \theta\left(q-k_{F \beta}\right)\right]^{2} .
\end{aligned}
$$

The pair-correlation functions also satisfy the the multicomponent version (applied to double-layer systems) of the Kimball relation ${ }^{19}$

$$
\lim _{r \rightarrow 0} g_{\alpha \beta}^{\uparrow \downarrow}(r)=-\lim _{q \rightarrow q} \frac{q^{3} e^{q d\left(1-\delta_{\alpha \beta}\right)}}{4 \pi e^{2} \mu_{\alpha \beta}\left(n_{\alpha} n_{\beta}\right)^{1 / 2}}\left[S_{\alpha \beta}^{\uparrow \downarrow}(q)-\delta_{\alpha \beta}\right] .
$$

\section{RESULTS AND DISCUSSION}

The integral equation for the interlayer effective interaction $I_{\alpha \beta}(q)$ can be classified as the Fredholm equation of the second kind. We solve Eq. (2) for electron-electron and electron-hole double-layer systems at equal density using matrix inversion techniques. In the previous applications ${ }^{7,10,16}$ the bare Coulomb interaction appearing in the kernel was further approximated to obtain an analytical solution to the integral equation. Using the scaled quantities, our results will appear as independent of the electron-hole

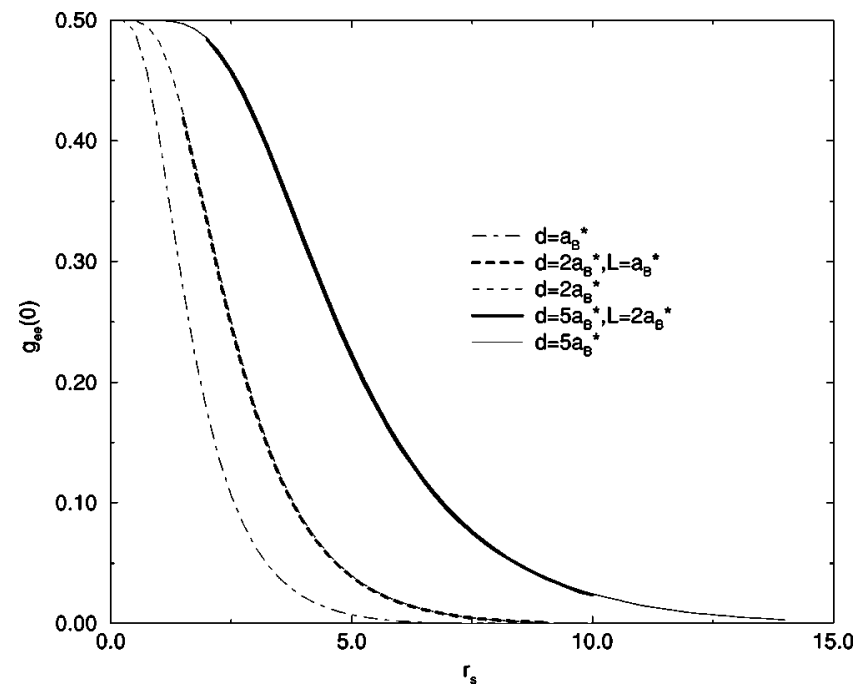

FIG. 1. The interlayer pair-correlation function at contact $g_{\text {ee }}(0)$ as a function of the layer density parameter $r_{s}$ for a double-layer electron system. The distance between the layers $d$, and the quantum-well thickness $L$ are indicated in the legend.

mass ratio $m_{e} / m_{h}$. All physical parameters are embodied in the definition of the effective Bohr radius $a_{B}^{*}$. We also note that in the examples given below, equal carrier densities are assumed for the layers.

\section{A. Electron-electron and electron-hole double layers}

In Fig. 1 we display the value of interlayer paircorrelation function at contact $g_{\text {ee }}(0)$ for a double-layer electron system as a function of the density parameter $r_{s}$. We observe that for a given interlayer separation $g_{\text {ee }}(0)$ decreases as the interaction strength (measured by $r_{s}$ ) increases. Unlike some other approximate theories, the ladder approximation results for $g_{\text {ee }}(0)$ always remains positive. Another noteworthy point here is that interlayer paircorrelation function $g_{\text {ee }}(0)$ is practically unaffected by the finite width of the layers. We have modeled the finite extent of the quantum wells using infinite square well model yielding a form factor

$$
F_{12}(q)=\frac{64 \pi^{4} \sinh ^{2}(x / 2)}{x^{2}\left(x^{2}+4 \pi^{2}\right)^{2}}
$$

where $x=q L, L$ being the width of the quantum wells. Thus the bare Coulomb interactions are modified so that $V_{12}(q)$ $\rightarrow V_{12}(q) F_{12}(q)$, and similarly for the intralayer interaction with corresponding form factor. In the case of intralayer interactions, finite width effects reduce the correlation effects and $g(0)$ increases ${ }^{13}$ with quantum-well width $L$. It turns out that the exponential factor $e^{-q d}$ in the interlayer interaction already limits the $q$-integration in Eq. (3) so that the finite width effects embodied in $F_{12}(q)$ are not very important. We shall see below that the situation somewhat changes when screening effects are considered.

Figure 2 shows the inverse of interlayer pair-correlation function at contact $g_{\text {eh }}^{-1}(0)$, for double-layer electron-hole 


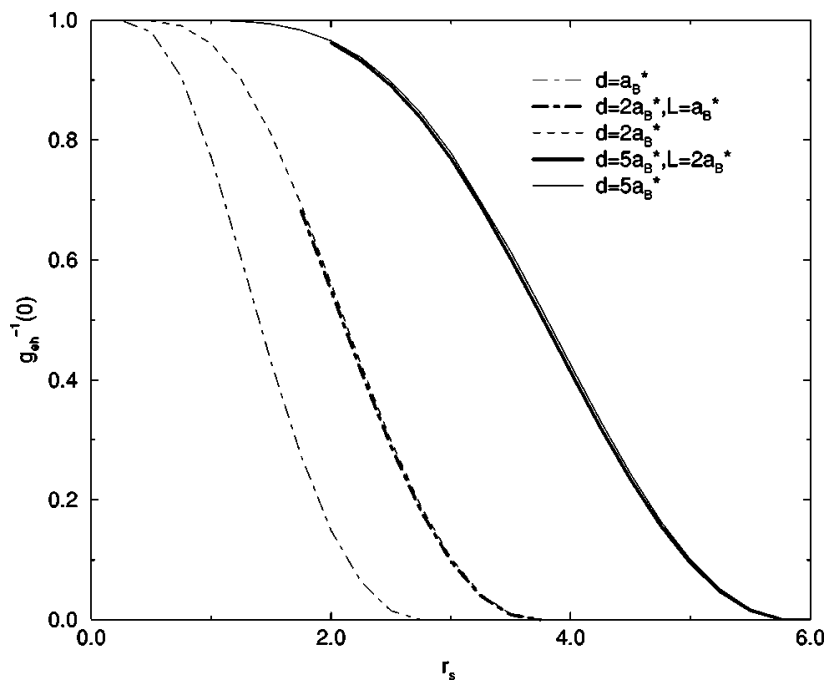

FIG. 2. The inverse of the interlayer pair-correlation function at contact $g_{\text {eh }}^{-1}(0)$ as a function of the layer density parameter $r_{s}$ for a double-layer electron-hole system. The distance between the layers $d$, and the quantum-well thickness $L$ are indicated in the legend.

systems as a function of $r_{s}$. We find that $g_{\text {eh }}(0)$ diverges at large $r_{s}$ for a given layer separation $d$. It is interesting, however, to note that $g_{\text {eh }}^{-1}(0)$ vanishes at some critical $r_{S}$ in a smooth way within the ladder approximation. In the estimates given by Liu, Swierkowski, and Neilson, ${ }^{3}$ the critical value of $r_{s}$ at which $g_{\text {eh }}^{-1}$ goes to zero was determined by extrapolation, and the density indicating an excitonic instability is sharply defined. In the ladder approximation, on the other hand, we are able to calculate $g_{\text {eh }}^{-1}(0)$ for any value of $d$ and $r_{s}$ for a more reliable prediction. We also find that $g_{\text {eh }}(0)$ is slightly affected by the finite thickness of the quantum wells, as indicated in Fig. 2. To better understand the transition to the excitonic state within the ladder approximation, we plot in Fig. 3 the $q$ dependence of the effective interlayer interaction $I_{\mathrm{eh}}(q)$ around the critical $r_{s}$. For a

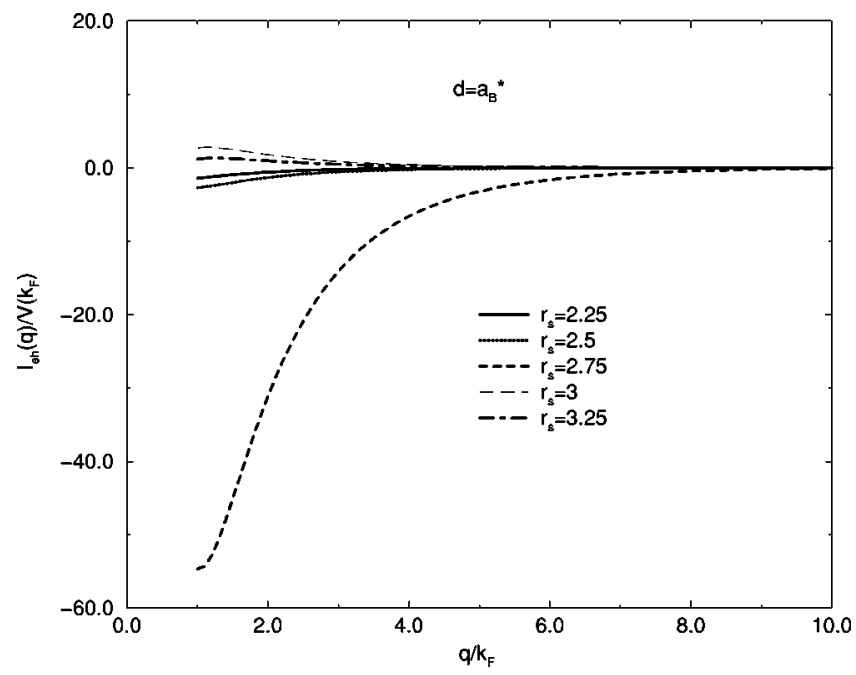

FIG. 3. The effective interaction $I_{\text {eh }}(q)$ as a function of $q$ for a double-layer electron-hole system at layer separation $d=a_{B}^{*}$ and various values of $r_{s}$.

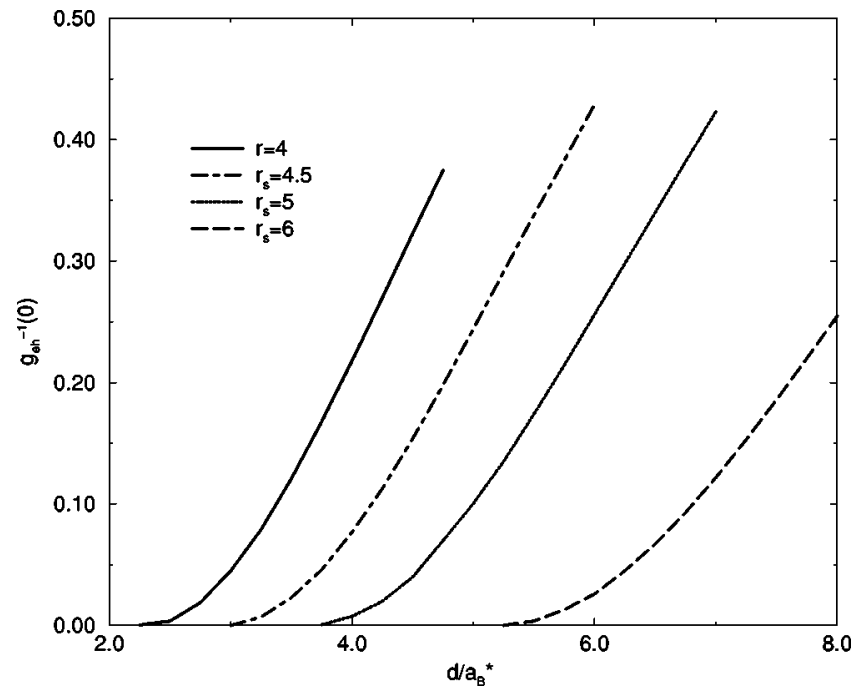

FIG. 4. The inverse of the contact pair-correlation function $g_{\text {eh }}^{-1}(0)$ for a double-layer electron-hole system as a function of the layer separation $d$. The values of the density parameter $r_{s}$ are indicated in the legend.

layer separation $d=a_{B}^{*}$, we observe that the vanishing of $g_{\text {eh }}^{-1}(0)$ arises from a small $q$ divergence in $I_{\mathrm{eh}}(q)$ at a critical density (i.e. $r_{s} \approx 2.75$ ). Figure 3 also shows that $I_{\mathrm{eh}}(q)$ changes its sign beyond the critical $r_{s}$. The critical behavior of $g_{\text {eh }}(0)$ is also seen in Fig. 4 where we display $g_{\text {eh }}^{-1}(0)$ as a function of $d$ for various values of $r_{s}$. The results shown in Fig. 4 verify that for a fixed carrier density, the electron-hole pair-correlation function diverges as the layer separation is decreased.

\section{B. Effects of screening}

We have seen in the previous section that $g_{\text {ee }}(0)$ and $g_{\text {eh }}(0)$ are hardly affected by the finite size of the quantumwells. When static screening effects are taken into effect the situation changes considerably. This is because in the screening function both the intralayer and interlayer bare Coulomb interactions come into play. Since the intralayer interactions are affected more by the finite width effects, we observe noticeable differences. Even in the case of zero-thickness layers because of the presence of intralayer interactions, screening effects modify the pair-correlation functions at contact. This was noted by Pugnaloni, Melgarejo, and Vericat $^{16}$ where they replace the bare interaction $V_{12}(q)$ by $V_{12}(q) / \varepsilon(q)$ in the kernel of the integral equation. In fact, they introduce a phenomenological momentum cutoff to modify the integral equation to account for screening. In this work we treat the screening effects within the static randomphase approximation and replace $V_{12}(q)$ by $V_{12}(q) / \varepsilon(q)$ in both the kernel and the inhomogeneous term in Eq. (1). Here $\varepsilon(q)$ is the static screening function of a double-layer electron-electron or electron-hole system given by

$$
\begin{aligned}
\varepsilon(q)= & {\left[1-\chi_{01}(q) V_{11}(q)\right]\left[1-\chi_{02}(q) V_{22}(q)\right] } \\
& -V_{12}^{2}(q) \chi_{01}(q) \chi_{02}(q),
\end{aligned}
$$




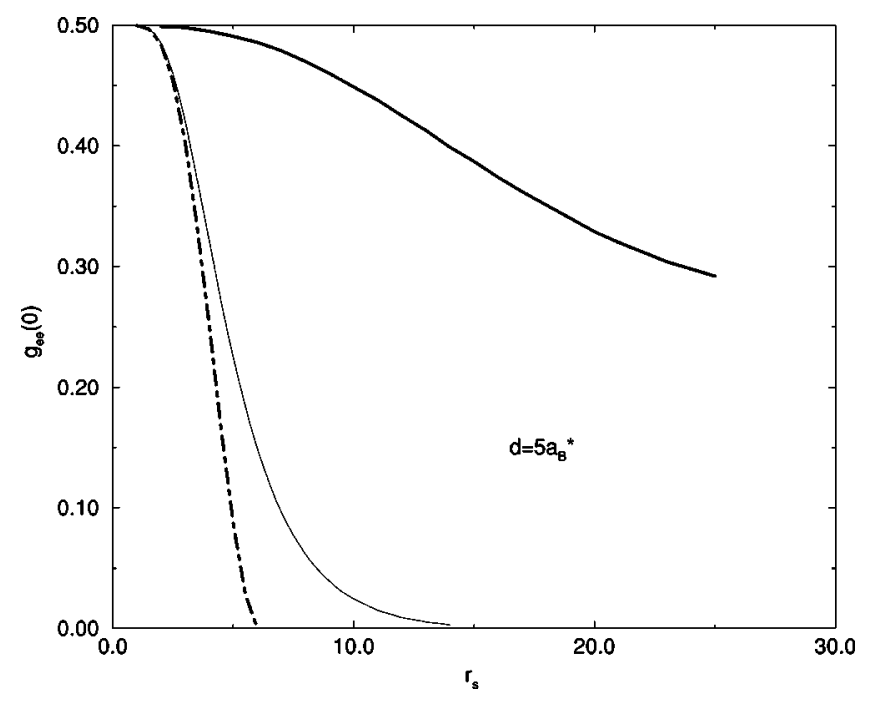

FIG. 5. The interlayer pair-correlation function $g_{\text {ee }}(0)$ as a function of $r_{s}$, at $d=5 a_{B}^{*}$. Thin solid and thick solid lines are for unscreened and screened ladder approximation results, respectively. If the screening is introduced only in the kernel the dot-dashed line results.

where $\chi_{0 \alpha}(q)$ are the static response functions for the noninteracting system in the layer $\alpha$.

In Fig. 5 we show the interlayer pair-correlation function in a double-layer electron system $g_{\text {ee }}(0)$ at contact for various approximations as a function of $r_{s}$. When the static screening effects are only introduced in the kernel of the integral equation [Eq. (1)] $g_{\text {ee }}(0)$ shows some departure from the unscreened case. In particular, the $r_{s}$ value at which $g_{\text {ee }}(0)$ becomes zero decreases. On the other hand, when the screening is taken into account in both the kernel and the inhomogeneous term in the integral equation the interlayer pair-correlation function $g_{\text {ee }}(0)$ shows a markedly different behavior. This latter way of including the screening effects is similar to the Lippmann-Schwinger equation treatment for the $T$-matrix in which the screened Coulomb interaction enters. $^{20,21}$ Similar results are displayed in Fig. 6 for the interlayer pair-correlation function $g_{\mathrm{eh}}^{-1}(0)$ in a double-layer electron-hole system. The inclusion of screening effects in the kernel mainly affects $g_{\text {eh }}^{-1}(0)$ at large $r_{s}$ values and a sharp divergence in $g_{\text {eh }}(0)$ at a critical $r_{s}$ is blurred. When a statically screened interaction in both the kernel and the inhomogeneous term is used, the resulting $g_{\text {eh }}(0)$ does not appear to show any instability in the range of $r_{s}$ values we investigate. Including the finite width effects only slightly lowers the curve $g_{\text {eh }}^{-1}(0)$, as can be seen in Fig. 6 .

Multicomponent generalization of the ladder approximation [in its local-approximation variant, i.e., $I_{\alpha \beta}(q)$ ] has found useful applications. For instance, the enhancement factor in the recombination rate of electron-hole plasmas as occur in photoexcited semiconductors is given in terms of the contact pair-correlation function $g_{\text {eh }}(0)$. Since $g_{\text {eh }}(0)$ within the ladder approximation can be reliably calculated, the results agree quite well with the experimental data. ${ }^{16,17}$ Similarly, the annihilation rate of positrons in an electronpositron two-component plasma is proportional to the

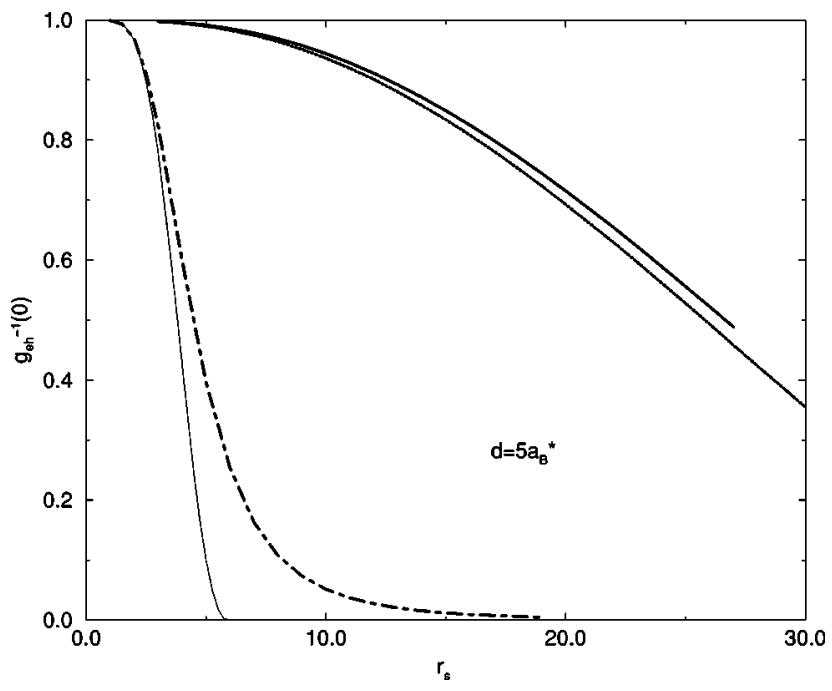

FIG. 6. The inverse of interlayer pair-correlation function $g_{\text {eh }}^{-1}(0)$ as a function of $r_{s}$, at $d=5 a_{B}^{*}$. Thin solid and thick solid lines are for unscreened and screened ladder approximation results, respectively. If the screening is introduced only in the kernel the dot-dashed line results. Dotted line indicates screened result with quantum-well width $L=a_{B}^{*}$.

contact pair-correlation function $g_{\text {ep }}(0)$. Although the selfconsistent field method of STLS has been analyzed ${ }^{20,22}$ to include multiple scattering effects and therefore, leading to a bound state instability, the positivity condition for the paircorrelation functions within this formalism is usually hard to fulfill. In the various applications of the ladder theory (including the multicomponent generalizations) the paircorrelation function remains positive for a large range of densities. We thus believe that the determination of instabilities such as exciton formation may be more reliably achieved within the ladder approximation.

\section{SUMMARY}

In summary, we have used the multicomponent generalization of the ladder approximation to study the correlation effects in double-layer electron-electron and electron-hole systems. Our short-range approximation regarding the effective interaction to be a local function of the momentum yields positive interlayer pair-correlation functions $g_{\text {ee }}(0)$ and $g_{\text {eh }}(0)$. This is an improvement over certain other approximate theories. Using the density dependence of $g_{\text {eh }}(0)$, we have estimated the stability of the system against exciton formation. We have found that the static screening effects significantly modify the contact values of interlayer paircorrelation functions in the ladder approximation.

\section{ACKNOWLEDGMENTS}

This work was partially supported by the Scientific and Technical Research Council of Turkey (TUBITAK) under Grant No. TBAG-2005, by NATO under Grant No. SfP971970, and by the Turkish Department of Defense under Grant No. KOBRA-001. We thank Dr. N. Bulut for useful discussions and E. Demirel for computational help. 
${ }^{1}$ See, for a recent review, C. B. Hanna, D. Haas, and J. C. DíazVélez, Phys. Rev. B 61, 13882 (2000).

${ }^{2}$ See, for a recent review, L. Świerkowski, D. Neilson, and J. Szymański, Aust. J. Phys. 46, 423 (1993).

${ }^{3}$ L. Liu, L. Świerkowski, and D. Neilson, Physica B 249-251, 594 (1998).

${ }^{4}$ K. S. Singwi, M. P. Tosi, R. H. Land, and A. Sjölander, Phys. Rev. 176, 589 (1968); K. S. Singwi and M. P. Tosi, Solid State Phys. 36, 177 (1981).

${ }^{5}$ L. J. Lantto, Phys. Rev. B 36, 5160 (1987).

${ }^{6}$ M. Alatalo, M. A. Salmi, P. Pietiläinen, and T. Chakraborty, Phys. Rev. B 52, 7845 (1995).

${ }^{7}$ H. Yasuhara, Solid State Commun. 11, 1481 (1972); J. Phys. Soc. Jpn. 36, 361 (1974).

${ }^{8}$ K. Awa, H. Yasuhara, and T. Asahi, Phys. Rev. B 25, 3670 (1982).

${ }^{9}$ D. N. Lowy and G. E. Brown, Phys. Rev. B 12, 2138 (1975).

${ }^{10}$ S. Nagano, K. S. Singwi, and S. Ohnishi, Phys. Rev. B 29, 1209
(1984).

${ }^{11}$ K. Takayanagi and E. Lipparini, Phys. Rev. B 54, 6122 (1996).

${ }^{12}$ N. Nafari and B. Davoudi, Phys. Rev. B 57, 2447 (1998).

${ }^{13}$ L. Calmels and A. Gold, Phys. Rev. B 57, 1436 (1998).

${ }^{14}$ D. L. Freeman, J. Phys. C 16, 711 (1983).

${ }^{15}$ F. Vericat and A. A. Melgarejo, Phys. Chem. Liq. 27, 235 (1994).

${ }^{16}$ L. A. Pugnaloni, A. A. Melgarejo, and F. Vericat, J. Phys.: Condens. Matter 11, 2607 (1999).

${ }^{17}$ A. A. Melgarejo, D. G. Renzi, C. O. Stoico, and F. Vericat, Physica E (Amsterdam) 3, 205 (1998).

${ }^{18}$ Y. Ousaka, H. Suehiro, and H. Yasuhara, J. Phys. C 18, 4471 (1985).

${ }^{19}$ J. C. Kimball, Phys. Rev. A 7, 1648 (1973).

${ }^{20}$ D. N. Lowy and A. D. Jackson, Phys. Rev. B 12, 1689 (1975).

${ }^{21}$ See, also, for a recent reference, D. O. Gericke, S. Kosse, M. Schanges, and M. Bonitz, Phys. Rev. B 59, 10639 (1999).

${ }^{22}$ A. Sjölander and M. J. Stott, Phys. Rev. B 5, 2109 (1972). 